\title{
Filter paper degradation by bacteria isolated from local termite gut.
}

\begin{abstract}
Bacterial strains isolated from the gut of the local termite Coptotermes curvignathus were inoculated into a buffered medium containing minerals and Whatman filter paper as the sole carbon source to observe the ability of the bacteria to digest solid substrate. The bacteria were Bacillus cereus strain Razmin A, Enterobacter aerogenes strain Razmin B, Enterobacter cloacae strain Razmin C, Acinetobacter strain Raminalimon and Chryseobacterium kwangyangense strain $\mathrm{Cb}$. The Gen Bank NCBI/EMBL accession numbers for the bacterial strains were EU294508, EU305608, EU305609, EU332791 and EU169201, respectively. The ability of bacterial cultures to grow in this medium as well as to digest the filter paper was determined by visual observation after 30 days. All bacterial cultures showed growth as the medium turned cloudy and the filter paper became macerated. Chryseobacterium kwangyangense strain $\mathrm{Cb}$ showed yellow pigmented colonies on the filter paper. Bacillus cereus strain Razmin A showed clumps of degraded filter paper with black dots.
\end{abstract}

Keyword: Cellulolytic bacteria; Termite gut; Filter paper degration. 